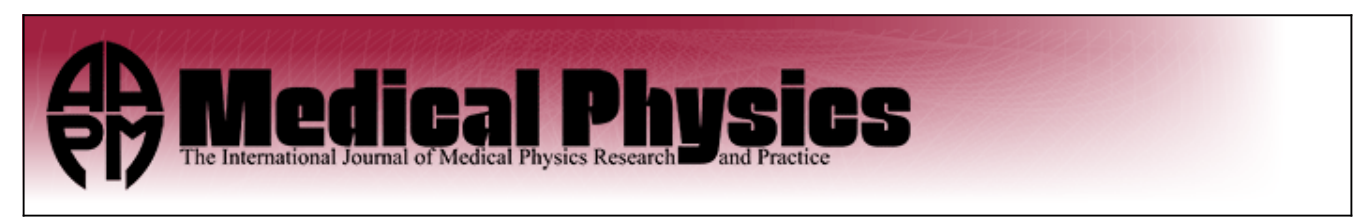

\title{
Towards 3D printed multifunctional immobilization for proton therapy: Initial materials characterization
}

Steven Michiels, Antoine D'Hollander, Nicolas Lammens, Mathias Kersemans, Guozhi Zhang, Jean-Marc Denis, Kenneth Poels, Edmond Sterpin, Sandra Nuyts, Karin Haustermans, and Tom Depuydt

Citation: Medical Physics 43, 5392 (2016); doi: 10.1118/1.4962033

View online: http://dx.doi.org/10.1118/1.4962033

View Table of Contents: http://scitation.aip.org/content/aapm/journal/medphys/43/10?ver=pdfcov

Published by the American Association of Physicists in Medicine

\section{Articles you may be interested in}

Technical Note: Immunohistochemical evaluation of mouse brain irradiation targeting accuracy with 3Dprinted immobilization device

Med. Phys. 42, 6507 (2015); 10.1118/1.4933200

Technical Note: Fabricating Cerrobend grids with 3D printing for spatially modulated radiation therapy: A feasibility study

Med. Phys. 42, 6269 (2015); 10.1118/1.4932223

3D printer generated thorax phantom with mobile tumor for radiation dosimetry

Rev. Sci. Instrum. 86, 074301 (2015); 10.1063/1.4923294

A deformable head and neck phantom with in-vivo dosimetry for adaptive radiotherapy quality assurance Med. Phys. 42, 1490 (2015); 10.1118/1.4908205

A method to evaluate dose errors introduced by dose mapping processes for mass conserving deformations Med. Phys. 39, 2119 (2012); 10.1118/1.3684951

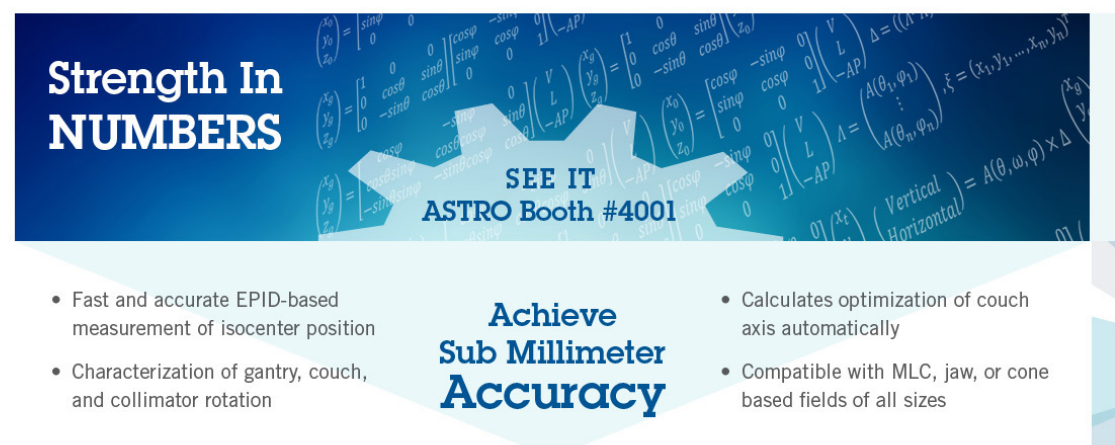

Version 6.5.X Is Here

Don't miss out on all the new leading edge features of The RIT Family of Products Version 6.5.X. Download it now! 


\title{
Towards 3D printed multifunctional immobilization for proton therapy: Initial materials characterization
}

\author{
Steven Michiels ${ }^{a)}$ \\ Department of Oncology, Laboratory of Experimental Radiotherapy, KU Leuven - University of Leuven, \\ Herestraat 49, Leuven 3000, Belgium \\ Antoine D'Hollander \\ Department of Medical Engineering, Materialise NV, Technologielaan 15, Haasrode 3001, Belgium \\ Nicolas Lammens and Mathias Kersemans \\ Department of Materials Science and Engineering, Ghent University, Technologiepark 903, \\ Zwijnaarde 9052, Belgium \\ Guozhi Zhang \\ Department of Radiology, KU Leuven - University of Leuven, Herestraat 49, Leuven 3000, Belgium \\ Jean-Marc Denis \\ Department of Radiotherapy and Oncology, Saint Luc University Clinics, Avenue Hippocrate 10, \\ Woluwe-Saint-Lambert 1200, Belgium \\ Kenneth Poels \\ Department of Radiation Oncology, University Hospitals Leuven, Herestraat 49, Leuven 3000, Belgium
}

\section{Edmond Sterpin}

Department of Oncology, Laboratory of Experimental Radiotherapy, KU Leuven - University of Leuven, Herestraat 49, Leuven 3000, Belgium and Université catholique de Louvain, Center of Molecular Imaging, Radiotherapy and Oncology, Institut de Recherche Expérimentale et Clinique, Avenue Hippocrate 54, Woluwe-Saint-Lambert 1200, Belgium

Sandra Nuyts, Karin Haustermans, and Tom Depuydt ${ }^{\text {b) }}$

Department of Oncology, Laboratory of Experimental Radiotherapy, KU Leuven - University of Leuven, Herestraat 49, Leuven 3000, Belgium and Department of Radiation Oncology, University Hospitals Leuven, Herestraat 49, Leuven 3000, Belgium

(Received 24 April 2016; revised 12 August 2016; accepted for publication 21 August 2016; published 7 September 2016)

Purpose: 3D printing technology is investigated for the purpose of patient immobilization during proton therapy. It potentially enables a merge of patient immobilization, bolus range shifting, and other functions into one single patient-specific structure. In this first step, a set of 3D printed materials is characterized in detail, in terms of structural and radiological properties, elemental composition, directional dependence, and structural changes induced by radiation damage. These data will serve as inputs for the design of 3D printed immobilization structure prototypes.

Methods: Using four different 3D printing techniques, in total eight materials were subjected to testing. Samples with a nominal dimension of $20 \times 20 \times 80 \mathrm{~mm}^{3}$ were $3 \mathrm{D}$ printed. The geometrical printing accuracy of each test sample was measured with a dial gage. To assess the mechanical response of the samples, standardized compression tests were performed to determine the Young's modulus. To investigate the effect of radiation on the mechanical response, the mechanical tests were performed both prior and after the administration of clinically relevant dose levels (70 Gy), multiplied with a safety factor of 1.4. Dual energy computed tomography (DECT) methods were used to calculate the relative electron density to water $\rho_{e}$, the effective atomic number $Z_{\text {eff }}$, and the proton stopping power ratio (SPR) to water SPR. In order to validate the DECT based calculation of radiological properties, beam measurements were performed on the 3D printed samples as well. Photon irradiations were performed to measure the photon linear attenuation coefficients, while proton irradiations were performed to measure the proton range shift of the samples. The directional dependence of these properties was investigated by performing the irradiations for different orientations of the samples.

Results: The printed test objects showed reduced geometric printing accuracy for 2 materials (deviation $>0.25 \mathrm{~mm}$ ). Compression tests yielded Young's moduli ranging from 0.6 to $2940 \mathrm{MPa}$. No deterioration in the mechanical response was observed after exposure of the samples to $100 \mathrm{~Gy}$ in a therapeutic MV photon beam. The DECT-based characterization yielded $Z_{\mathrm{eff}}$ ranging from 5.91 to 10.43. The SPR and $\rho_{e}$ both ranged from 0.6 to 1.22 . The measured photon attenuation coefficients at clinical energies scaled linearly with $\rho_{e}$. Good agreement was seen between the DECT estimated 
SPR and the measured range shift, except for the higher $Z_{\text {eff }}$. As opposed to the photon attenuation, the proton range shifting appeared to be printing orientation dependent for certain materials.

Conclusions: In this study, the first step toward 3D printed, multifunctional immobilization was performed, by going through a candidate clinical workflow for the first time: from the material printing to DECT characterization with a verification through beam measurements. Besides a proof of concept for beam modification, the mechanical response of printed materials was also investigated to assess their capabilities for positioning functionality. For the studied set of printing techniques and materials, a wide variety of mechanical and radiological properties can be selected from for the intended purpose. Moreover the elaborated hybrid DECT methods aid in performing in-house quality assurance of 3D printed components, as these methods enable the estimation of the radiological properties relevant for use in radiation therapy. (C) 2016 American Association of Physicists in Medicine. [http://dx.doi.org/10.1118/1.4962033]

Key words: patient immobilization, dual energy CT, 3D printing, proton therapy, bolus

\section{INTRODUCTION}

The continuous improvement of radiation therapy techniques mainly aims at improved local tumor control with reduced side effects of the treatment. In this pursuit, the use of protons is highly promising due to their advantageous depth dose profile. The finite range and the sharp fall-off of the dose deposition in the Bragg peak enable a potentially better target dose conformity and normal tissue sparing compared to photons. The accurate dose deposition control of proton pencil beam scanning, however, comes with an increased sensitivity to various range and setup uncertainties. ${ }^{1-3}$ As these uncertainties may cause the delivered dose to considerably deviate from the planned dose, effectively dealing with them is the biggest challenge in realizing the full potential of proton therapy (PT). ${ }^{4}$ In an effort to increase the PT treatment effectiveness for head-and-neck cancer (HNC), for which intensity-modulated proton therapy (IMPT) is an attractive modality, ${ }^{5,6}$ novel solutions using $3 \mathrm{D}$ printing technology are proposed.

3D printing or additive manufacturing is an appealing technique as it offers a high degree of flexibility in object shaping from a wide range of materials. Large-scaled printers with print bed side lengths of a few meters require a considerable capital investment, but desktop sized printers at entry-level prices have become readily available on the market. A variety of printing techniques exist, which allows to exploit each technique's relative strengths in function of the envisioned application. Fused deposition modeling (FDM), for example, can be used to reduce costs in the industrial manufacturing of components, such as lightweight airplane parts. Polyjet ${ }^{\mathrm{TM}}$ (PJ) or stereolithography (SLA) can be selected for applications where the highest achievable printing accuracy is required, such as in the fabrication of patient-specific implants ${ }^{7}$ or in the 3D model creation for complex surgery planning. ${ }^{8}$ Selective laser sintering (SLS) allows to produce heat-resistant metallic components in complex shapes, for example, for automotive engine parts. 3D printing in general is increasingly investigated for use in radiotherapy, for the manufacturing of, amongst others, electron bolus, proton range compensators, and range modulator wheels. ${ }^{9-11}$ The technology has been considered as well for the construction of anthropomorphic phantoms for dosimetry purposes. ${ }^{12}$ We aim to use $3 \mathrm{D}$ printing to manufacture a customized immobilization device fitted to the patient's body outlines.

Such a 3D printed device could yield improvements in the accuracy and reproducibility of patient positioning and immobilization during treatment. The current practice of thermoplastic masks can encompass significant interfractional variation in the positioning of structures. ${ }^{13,14}$ Also, substantial changes occur in the head-and-neck anatomy during the treatment course, with potentially a detrimental influence on the quality of the immobilization and hence on the delivered dose distribution. ${ }^{15}$ Lastly, certain types of thermoplastic masks are known to shrink after the initial application, which can reduce the patient-specificity and can cause patient discomfort. In this regard, the ability to use different 3D printed materials with different degrees of elasticity in patientspecific shapes would enable to pursue a balance between accurate, reproducible immobilization, and patient comfort. Moreover, a modular approach of the design would allow to reprint substructures in function of the anatomical changes, aiding in implementing an adaptive immobilization.

3D printing could also yield a number of additional treatment enhancements, such as the integration of range shifter (RS) in the immobilization device for the treatment of superficial lesions, i.e., as bolus. The attachment of RS to the nozzle, namely, causes the presence of an air gap between the nozzle and the patient skin, which is not properly modeled by the current generation of pencil beam dose calculation algorithms. ${ }^{16}$ Moreover the air gap length is known to have a strong impact on the lateral dose fall-off of proton beams due to the angular spread created in the RS, ${ }^{17-19}$ with negative dosimetric consequences. Integrating RS as bolus in the 3D printed device itself would solve this issue, as the air gap would be completely eliminated. Moreover, such an integrated solution would allow a safer clearance between moving gantry parts and patient, eliminating collision risk and allowing for a more time-efficient treatment delivery due to the omission of hardware movements. Lastly, the degrees of freedom in the generated shapes offered by 3D printing could allow to integrate other functionalities besides bolus. In vivo dosimetry with a high positional accuracy of detectors relative to the 
patient anatomy, for instance, comes into reach, which could live up to the strong demand for patient-specific dosimetry and quality assurance (QA) techniques in IMPT.

Given that the intended device has both a mechanical and radiological functionality, in a first step, a broad characterization of possible 3D printed materials and techniques is to be carried out, which is the scope of this paper. Due to the discrete nature of the printing process, a different overall behavior of 3D printed materials could be expected compared to the same materials produced in a more established manner. Topics such as dimensional accuracy, mechanical elasticity including its potential change induced by radiation, and interaction with protons and photons are covered. Moreover, successful clinical implementation of a device consisting of $3 \mathrm{D}$ printed supporting and beam modifying structures requires reliable material assessment methods that fit within the clinical workflow. Therefore, dual-energy computed tomography (DECT) methods for the determination of radiological properties are elaborated and applied to 3D printed materials for the first time. These methods are verified as well by photon attenuation and proton range shift measurements.

\section{MATERIALS AND METHODS}

\section{A. 3D printing technologies and materials}

In total eight materials were subjected to testing in this work, using four different printing techniques. An overview of the considered printing techniques and materials is given in Table I. The FDM process is based on the heating and layering of thermoplastics. A temperature-controlled extrusion head is fed with thermoplastic material which is heated to a semiliquid state. The head extrudes and directs the material layer by layer onto a fixtureless base. After being deposited, the material cools down and hardens. The result of the solidified material laminating to the preceding layer is a plastic 3D model built up one layer at a time. The filling degree of the printed object can be varied freely in this technique. As it takes time for the thermoplastic to harden and for the layers to bond together, the use of support structures is required when generating objects with overhanging geometries. An advantage of FDM is that dual-nozzle configurations exist, which allow to create these support structures in water-soluble material. In general this printing technique is the cheapest and most accessible one, but is also the least capable in terms of printing speed, accuracy, and surface finish.

The other techniques use a slice by slice technique similar to FDM, but require exposure to laser light for the material to harden or "cure." The SLA process uses a tank of liquid polymer resin in which the layers are cured and solidified by an UV laser. In general, SLA provides the best accuracy and surface finish. Support structures, however, are to be removed by hand, which tends to be time-consuming. This printing technique is mostly used for building large parts or parts where different finishing degrees are required. The PJ technique simultaneously jets multiple photopolymer materials onto a layer on a build tray, after which the layer is immediately cured by an UV laser. At the expense of the highest cost, this technique offers multimaterial capabilities with fine detail. A trade-off, however, is that exposure of the materials to ambient heat or humidity can cause dimensional changes. Also these materials in general have limited mechanical properties. Lastly, in the SLS technique powder-based materials are used with particle size in the order of magnitude of $50 \mu \mathrm{m}$. Successive powder layers are spread on top of each other on the build platform. After deposition, the particles are selectively bound together by the elevation of the powder temperature due to the exposure to a $\mathrm{CO}_{2}$ laser beam. This technique offers the possibility to print metallic materials as well. In addition, no support structures are needed as support is provided by the print bed itself, which allows to print complex shapes. On the downside, because of the elevated temperatures required for SLS, cooling times can be considerable. Also the use of particles result in rather rough surfaces.

\section{B. Printing of test object samples}

In order to perform the different characterization methods, two identical test objects were printed for each of the eight materials. A specified dimension of $20 \times 20 \times 80 \mathrm{~mm}^{3}$ was used. The actual dimensions of each sample were measured with a dial gage (model: 2046S-Mitutoyo, Kawasaki) with a spatial resolution of $10 \mu \mathrm{m}$, and compared with the specified nominal values. The distance between the $20 \times 20 \mathrm{~mm}^{2}$ faces was measured at one central point. The distance between the $20 \times 80 \mathrm{~mm}^{2}$ faces was measured at six points distributed equally over the surface.

TABLE I. Overview of the considered printing techniques and materials. The manufacturer-quoted maximum part dimensions (Ref. 20), standard accuracy, printing process layer thickness, and used materials are displayed.

\begin{tabular}{lcccc}
\hline \hline & \multicolumn{4}{c}{ Production technique } \\
\cline { 2 - 5 } Property & FDM & SLA & PJ & SLS \\
\hline Max. dimensions $\left(\mathrm{mm}^{3}\right)$ & $914 \times 610 \times 914$ & $2100 \times 700 \times 800$ & $500 \times 400 \times 200$ & $650 \times 330 \times 560$ \\
Standard accuracy $(\mu \mathrm{m})$ & \pm 200 & \pm 150 & $\pm 100-200$ & \pm 300 \\
Layer thickness $(\mu \mathrm{m})$ & $100-300$ & $50-200$ & $16-32$ & $60-180$ \\
Materials & ABS & TuskXC2700T & TangoPlus & PolyAmide (PA)-12 \\
& & & VeroWhite & PA-alumide \\
& & & PA-glass fiber \\
& & & TPU \\
\hline \hline
\end{tabular}




\section{C. Mechanical response measurements}

In order to assess the mechanical response of the 3D printed samples, compression tests were performed to determine the Young's modulus (Sec. 2.C.1). To investigate the effect of radiation on the mechanical response, the compression tests were performed both prior and after the administration of clinically relevant dose levels, multiplied with a safety factor (Sec. 2.C.2).

\section{C.1. Compression tests-Young's modulus}

The samples were compression tested according to the ASTM D695 standard ${ }^{21}$ in a tensile testing machine (model: 5800R-Instron, Norwood, MA). Three linear variable differential transformers were used to measure the displacement between top and bottom faces of the compression plates. To avoid plastic deformation of the samples, the applied strain levels were kept as low as possible. For the stiff materials ( $>100 \mathrm{MPa}$ ), the compressive strain level was limited to $0.5 \%$ and the loads were recorded with a $100 \mathrm{kN}$ load cell. For the flexible materials $(<100 \mathrm{MPa})$, a compressive strain of $5 \%$ was applied and a $1 \mathrm{kN}$ load cell was used in order to obtain a more accurate measurement.

\section{C.2. Photon irradiation-Radiation impact}

Irradiation of the samples was done in a clinical photon beam of $6 \mathrm{MV}$, which was calibrated to deliver $0.8 \mathrm{~Gy} / 100$ monitor units (MU) at a depth in water of $10 \mathrm{~cm}$ (source-tosurface distance SSD $=90 \mathrm{~cm}$ ). To each sample positioned at a depth in solid water of $1 \mathrm{~cm}$ using the same SSD, 1000 MU were delivered, yielding a delivered dose of roughly $100 \mathrm{~Gy}$. This corresponds to a typical target dose of $70 \mathrm{~Gy}$ to a HNC boost volume, elevated with a safety factor of around 1.4.

\section{D. Radiological properties calculations based on DECT data}

DECT-methodologies were used to calculate various radiological properties of the samples. In order to verify the deployed DECT characterization methods, tissue surrogate reference rods (RMI 467 Electron Density Phantom, Gammex, Middleton) and a plastic water (PW-CIRS, Inc., Norfolk) rod with a known elemental composition were included in the set of scanned samples (Sec. 2.D.1). The CT acquisition method is described in Sec. 2.D.2. The relative electron density to water $\rho_{e} \equiv \rho_{e, \text { medium }} / \rho_{e \text {,water }}$ (Sec. 2.D.3), the effective atomic number $Z_{\text {eff }}$ (Sec. 2.D.4), and the proton stopping power ratio (SPR) to water SPR (Sec. 2.D.5) were calculated from the acquired images.

\section{D.1. Reference samples and basic formulae}

The composition and the properties of the reference materials are provided in the supplementary material. ${ }^{22,23}$ The relative electron density to water $\rho_{e}$ of each reference material was determined from its electron density per volume $\rho_{e, \text { medium }}=N_{A} \rho \sum_{i} w_{i} Z_{i} / A_{i}$, with $w_{i}$ the mass fraction weight of element $i$. The effective atomic number was calculated ${ }^{24,25}$ as $Z_{\text {eff }}=\left(\sum_{i} \alpha_{i} Z_{i}^{m}\right)^{1 / m}$, where a value of 3.1 for the exponent $m$ was adopted from Hünemohr et $a l .{ }^{26}$ as the same scanner spectra were used in this work. The proton stopping power ratio to water SPR was calculated using the simplified ${ }^{27}$ Bethe-Bloch formula

$$
\mathrm{SPR}=\rho_{e} \cdot f\left(\beta c, I_{m, \text { medium }}, I_{m, \text { water }}\right)
$$

where $\beta c$ is the velocity of the proton, and $I_{m}$ is the mean ionization energy ${ }^{23}$ of the considered medium. To put the calculations in line with the experiments (see Sec. 2.E.2), a value of 0.347 was taken for $\beta$, which corresponds to protons with a kinetic energy of $62 \mathrm{MeV}$.

\section{D.2. DECT image acquisition}

The reference samples as well as the $3 \mathrm{D}$ printed test samples under characterization were scanned with a dual source CT scanner (model Somatom Definition FLASHSiemens Medical, Forchheim). One sample at a time was inserted in a cavity on the inner circle of a RMI 467 phantom. The dual energy mode applied 80 and $140 \mathrm{kVp}$, the latter with additional Sn filtration to improve spectrum separation. A tube current ratio of 360/148 mAs was used. The image reconstruction was done with a clinically used abdomen protocol with a D30f convolution kernel and using a $2 \mathrm{~mm}$ slice thickness. Scans of the reference samples were taken in single energy mode as well, with a tube voltage of $120 \mathrm{kVp}$. For each volume of interest (VOI), the inner $90 \%$ of the voxels was used to extract the average number of Hounsfield units (HU). For the single energy scan, the standard deviation, skewness, and kurtosis of the CT-numbers distribution were extracted as well.

\section{D.3. Calculation of $\rho_{e}$}

The extraction of $\rho_{e}$ from the DECT scans was done using the $\Delta \mathrm{HU}-\rho_{e}$ conversion method from Saito. ${ }^{28}$ In this procedure, the weighted subtraction of the CT numbers measured at two different energies was introduced, $\Delta \mathrm{HU}$ $\equiv(1+\alpha) \mathrm{HU}_{H}-\alpha \mathrm{HU}_{L}$, where $\mathrm{HU}_{H}$ and $\mathrm{HU}_{L}$ are, respectively, the CT numbers in $\mathrm{HU}$ at the high and low $\mathrm{kVp}$, and $\alpha$ is a scanner-specific weighting factor for the subtraction. A linear function for the calibrated $\rho_{e}{ }^{\mathrm{cal}}$ was then fitted to the dataset of $\rho_{e}$ and $\Delta \mathrm{HU}(\alpha)$ for the reference materials,

$$
\rho_{e}^{\mathrm{cal}}=a_{1} \frac{\Delta \mathrm{HU}(\alpha)}{1000}+a_{0},
$$

where the final value for the parameter $\alpha$ was optimized to yield the largest coefficient of determination $R^{2}$. The found correlation was finally used to calculate the $\rho_{e}$ for the 3D printed materials with unknown elemental composition from the DECT data. 


\section{D.4. Calculation of $Z_{\text {eff }}$}

Effective atomic numbers were extracted from DECT images using the tissue substitute method (TSM) from Landry et $a l^{29}$ This method employs the Rutherford parameterization $^{30}$ of the linear attenuation coefficients to derive a correlation (3) between $Z_{\text {eff }}$ and the ratio of the linear attenuation coefficients measured at the low and high $\mathrm{kVp}$ of the CT scanner:

$$
\begin{aligned}
\frac{\mu_{80 \mathrm{kVp}} / \mu_{80 \mathrm{kV}, \text { water }}}{\mu_{140 \mathrm{kVp}} / \mu_{140 \mathrm{kVp}, \text { water }}} & \left.\equiv \mu\right|_{140 \mathrm{kVp}} ^{80 \mathrm{kVp}} \\
& \approx \frac{1+C_{80 \mathrm{kVp}} Z_{\text {eff }}^{m-1}}{A_{140 \mathrm{kVp}}+C_{140 \mathrm{kVp}} Z_{\text {eff }}^{m-1}},
\end{aligned}
$$

where the fit parameters $m, C_{80 \mathrm{kVp}}, A_{140 \mathrm{kVp}}$, and $C_{140 \mathrm{kVp}}$ are constrained to positive values, and where the inversed function can be expressed analytically:

$$
Z_{\mathrm{eff}}=\left(\frac{1-\left.\mu\right|_{140 \mathrm{kVp}} ^{80 \mathrm{kV} p} A_{140 \mathrm{kVp}}}{\left.\mu\right|_{140 \mathrm{kVp}} ^{80 \mathrm{kV} p} C_{140 \mathrm{kVp}}-C_{80 \mathrm{kVp}}}\right)^{\frac{1}{m-1}}
$$

The theoretical $Z_{\text {eff }}$ was calculated as described in Sec. 2.D.1. The TSM then consisted in fitting (3) to the calculated $Z_{\text {eff }}$ and $\mu^{80 / 140 \mathrm{kV}}$ for the reference materials. Equation (4) then enabled to estimate $Z_{\text {eff }}$ for the 3D printed materials as well.

\section{D.5. Calculation of SPR}

The equations described in Sec. 2.D.1 allow to determine the proton SPR of the known reference materials using their elemental composition. For the 3D printed materials, however, the elemental composition is not always known or available. Therefore the formalism presented in Secs. 2.D.3 and 2.D.4 to calculate $\rho_{e}$ and $Z_{\text {eff }}$ using DECT data was used to estimate their SPR as well. For the missing information, $I_{m}$, a parameterization ${ }^{26}$ through $Z_{\mathrm{eff}}$ from 71 tissue surrogate compositions was used as follows:

$$
\ln I_{m}=a Z_{\mathrm{eff}}+b,
$$

where the parameter couple $(a, b)$ has a value of $(0.125$, $3.378)$ for $Z_{\mathrm{eff}}<8$ and $(0.098,3.376)$ for $Z_{\mathrm{eff}}>9$. Through the combination of (1) and (5), the SPR can then be approximated by a function of the estimated $\rho_{e}$ and $Z_{\text {eff }}$ as follows:

$$
\mathrm{SPR} \approx \rho_{e} \frac{11.73-\left(a Z_{\mathrm{eff}}+b\right)}{7.41} .
$$

\section{E. Beam measurements}

In order to validate the DECT based calculation of radiological properties, beam measurements were performed on the 3D printed samples. Photon irradiations were performed in order to measure the photon linear attenuation coefficients (Sec. 2.E.1) of the samples. Proton irradiations were performed in order to measure their proton range shift (Sec. 2.E.2). As a means to estimate the accuracy of the performed measurements, one sample with a known photon attenuation coefficient and proton range shift, namely, a block of PW with the same nominal dimensions, was included in the set of irradiated samples.

\section{E.1. Photon attenuation measurements}

Photon irradiations were performed in clinical photon beams of 6 and $10 \mathrm{MV}$ to measure the photon linear attenuation coefficients of the samples. A narrow beam geometry with a PW miniphantom as proposed by Georg and Dutreix ${ }^{31}$ was used to ensure that the primary beam alone is considered and that the influence of scattered rays from the head and from the attenuating material is excluded as much as possible. The dose rate was measured while inserting samples with different thicknesses in the beam. Fitting the measured data to an exponential curve allowed to determine the spectrum averaged attenuation coefficients $\mu_{\mathrm{tr}}$.

\section{E.2. Proton range shift measurements}

Range shift measurements of the samples were performed at the Cyclotron Resources Center of the Universite catholique de Louvain (Louvain-la-Neuve, Belgium), in a $62 \mathrm{MeV}$ nonmodulated broad proton beam. A $20 \mathrm{~mm}$ diameter brass aperture was used as a final collimator. Radiochromic film (type Gafchromic EBT-XD-Ashland, Inc., Covington) was sandwiched between two CIRS PW plates, with the film edge matched to the proximal surface of the plastic plates. The stack was positioned in line with the beam axis, but with a tilt angle $\theta$ of $3^{\circ}$ (verified with a digital level) in order to minimize the impact of the difference in proton stopping power between the interfacing media. ${ }^{32}$ The distance between the collimator and the $\mathrm{PW}$ edge was $15 \mathrm{~cm}$. Bragg peaks were acquired as such (see Fig. 1).

To determine the realized range shifts, Bragg peaks were acquired as well with the 3D printed samples inserted between the collimator and the stack. The samples were positioned with the $2 \mathrm{~cm}$ nominal length sides parallel to the beam axis. The samples were pressed against the stack edge in order to avoid any air gap. To measure the orientation dependency of the range shift due to the discrete $3 \mathrm{D}$ printing manufacturing process, irradiations were performed for two orthogonal orientations of the samples.

RGB 48-bit scans of the irradiated films were acquired using a flatbed scanner (model 10000XL-Epson, Suwa). Precalibration of the films using the method developed by Crijns et al..$^{33}$ prior to the proton beam measurements allowed to convert the films scans to dose maps. Three depth dose

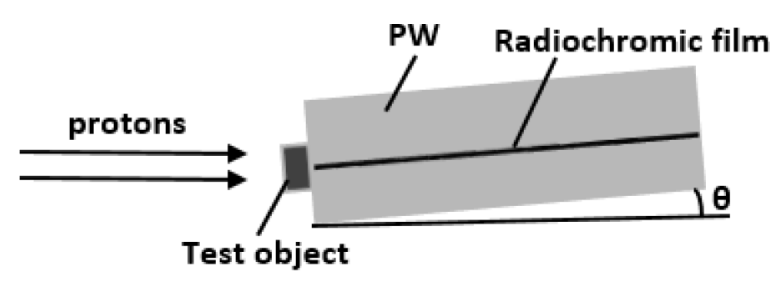

Fig. 1. Tilted setup of the radiochromic film sandwiched between PW plates. 
profiles were extracted from the central region of each broad Bragg peak and averaged out. The true depth dose curve was finally obtained by a $1 / \cos \theta$ scaling of the averaged depth dose curve to correct for the $3^{\circ}$ tilt of the setup.

The range of each Bragg peak was determined by normalizing the profiles to the maximum dose and by taking the distal $80 \%$ position $\left(d_{80}\right)$ of the peak ${ }^{34}$ relative to the surface/edge of the film. The range shift realized by any of the materials inserted was determined by subtracting the $d_{80}$ with the material inserted from the $d_{80}$ without any material inserted. This measured range shift was compared with the expected range shift as calculated by the DECT estimated SPR (Sec. 2.D.5),

$$
\Delta R_{80, \text { expected }}=t_{\text {sample }} \frac{\mathrm{SPR}_{\text {medium to water }}}{\mathrm{SPR}_{\mathrm{PW} \text { to water }}},
$$

where $t_{\text {sample }}$ represents the measured sample thickness traversed by the protons, $\mathrm{SPR}_{\text {medium to water }}$ represents the stopping power ratio to water as estimated before and where

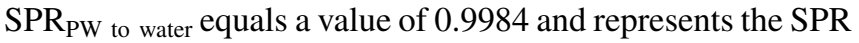
of PW to water, which compensates for the measurements being performed in PW instead of in real water.

In order to quantify the uncertainty on the range shift determination, the aforementioned procedure was performed six times for a sample of PW with the same nominal dimensions.

\section{RESULTS}

\section{A. Geometrical printing accuracy}

Table II provides a summary of the results from the dial gage measurements performed on the 3D printed samples. TangoPlus, which was the most flexible materials from the set, was the only material with an average thickness value below the lower limit. It exhibited a significant variation of the thickness over the surface as well, which resulted in a visible curvature of the surfaces. VeroWhite, which was made using the same printing technique (PJ) but had a significantly higher stiffness (see Sec. 3.B), did not show any out of tolerance dimensions. All measured dimensions, both thickness and length, of the TPU samples were above their upper limit. This resulted in an average deviation in thickness and in length of more than $0.5 \mathrm{~mm}$. The other laser sintered materials did not show out of tolerance behavior, except for one measured length for the PA-12 samples. Tusk, the only material printed by stereolithography, had all length measurements out of tolerance.

\section{B. Mechanical response}

Table III shows the Young's modulus prior and after irradiation as measured in the compression test. Initial Young's moduli as low as 0.6 MPa were seen for TangoPlus. VeroWhite, which was also printed with the PJ technique, exhibited a significantly higher stiffness, indicating that significantly different degrees of stiffness could be obtained using the same printing technique. In the group of laser sintered materials, the influence of additives such as alumide grains or glass fibers to PolyAmide was clearly seen, with Young's moduli observed up to $2940 \mathrm{MPa}$. The results suggest that most materials experienced a stiffening effect due to exposure to radiation.

\section{C. Radiological properties calculations based on DECT data}

\section{C.1. CT numbers}

The CT numbers of the scans of the reference materials and the 3D printed samples are given in the supplementary material. $^{22}$ An overview of the DECT based calculated radiological properties is given as well. The standard deviations on the CT-number distribution of the 3D printed samples were of roughly the same magnitude as the reference samples, except for ABS, PA-Alu, and to a lesser extent PAGF, which show higher standard deviations. The skewness and kurtosis are notably higher for ABS and VeroWhite than for the other samples.

TABLE II. Average and standard deviation of the centrally measured thickness, together with the range over which the thickness and length span over the complete surfaces of the different 3D printed samples. The shown tolerances represent the worse of the manufacturer-quoted tolerances. The nominal dimensions are $20 \times 20 \times 80 \mathrm{~mm}^{3}$.

\begin{tabular}{|c|c|c|c|c|c|c|c|}
\hline \multirow[b]{3}{*}{$\begin{array}{l}\text { Material (printing } \\
\text { technique) }\end{array}$} & \multirow[b]{3}{*}{$\begin{array}{l}\text { Tolerance } \\
(\mathrm{mm})\end{array}$} & \multicolumn{4}{|c|}{ Thickness } & \multirow{2}{*}{\multicolumn{2}{|c|}{$\begin{array}{l}\text { Length } \\
\text { Central }\end{array}$}} \\
\hline & & \multicolumn{2}{|r|}{ Central } & \multicolumn{2}{|c|}{ Overall } & & \\
\hline & & $\begin{array}{l}\text { Average } \\
(\mathrm{mm})\end{array}$ & $\begin{array}{c}\text { Standard deviation } \\
(\mathrm{mm})\end{array}$ & $\begin{array}{l}\text { Min } \\
(\mathrm{mm})\end{array}$ & $\begin{array}{l}\operatorname{Max} \\
(\mathrm{mm})\end{array}$ & $\begin{array}{l}\text { Min } \\
(\mathrm{mm})\end{array}$ & $\begin{array}{l}\text { Max } \\
(\mathrm{mm})\end{array}$ \\
\hline ABS (FDM) & 0.2 & 20.18 & 0.19 & 19.99 & 20.42 & 79.95 & 80.00 \\
\hline Tusk (SLA) & 0.15 & 19.88 & 0.10 & 19.77 & 19.98 & 80.58 & 80.85 \\
\hline TangoPlus (PJ) & 0.2 & 19.39 & 0.25 & 18.8 & 19.7 & 78.55 & 79.85 \\
\hline VeroWhite (PJ) & 0.2 & 20.05 & 0.08 & 19.98 & 20.15 & 80.02 & 80.2 \\
\hline PA-12 (SLS) & 0.3 & 20.03 & 0.06 & 19.84 & 20.1 & 79.95 & 80.85 \\
\hline PA-Alu (SLS) & 0.3 & 20.12 & 0.06 & 20 & 20.25 & 80.11 & 80.35 \\
\hline PA-GF (SLS) & 0.3 & 20.09 & 0.14 & 19.94 & 20.22 & 80.1 & 80.28 \\
\hline TPU (SLS) & 0.3 & 20.63 & 0.16 & 20.4 & 20.79 & 80.58 & 80.65 \\
\hline
\end{tabular}


TABLE III. Young's modulus measured according to ASTM D695 compression test of the 3D printed samples before and after irradiation with $100 \mathrm{~Gy}$. The shown measurement uncertainties depend on the load cell capacity, the Young's modulus, and the applied strain.

\begin{tabular}{lccccc}
\hline \hline & \multicolumn{3}{c}{ Young's modulus } \\
\cline { 2 - 6 } & & & & \multicolumn{2}{c}{ Uncertainty } \\
\cline { 2 - 6 } Material (Printing & $\begin{array}{c}\text { Prior irradiation } \\
\text { technique) }\end{array}$ & $\begin{array}{c}\text { After irradiation } \\
(\mathrm{MPa})\end{array}$ & $\begin{array}{c}\text { Relative difference } \\
(\%)\end{array}$ & $\begin{array}{c}\text { Relative } \\
(\%)\end{array}$ & $\begin{array}{c}\text { Absolute } \\
(\mathrm{MPa})\end{array}$ \\
\hline ABS (FDM) & 629 & 655 & 4.1 & 5.0 & 31 \\
Tusk (SL) & 1874 & 2200 & 17.4 & 1.3 & 25 \\
TangoPlus (PJ) & 0.6 & 0.6 & 0 & 5.2 & 0.031 \\
VeroWhite (PJ) & 1981 & 2450 & 23.7 & 1.1 & 21 \\
PA-12 (SLS) & 1633 & 1800 & 10.2 & 1.5 & 25 \\
PA-Alu (SLS) & 2940 & 3445 & 17.2 & 0.71 & 21 \\
PA-GF (SLS) & 2705 & 3017 & 11.5 & 0.92 & 25 \\
TPU (SLS) & 33 & 34 & 3.0 & 0.95 & 0.31 \\
\hline \hline
\end{tabular}

\section{C.2. Relative electron density $\rho_{e}$}

For the reference materials, a value of 0.0041 for the standard error of the estimate $\left(S_{\text {est }}\right)$ was obtained for $\rho_{e}$. The maximum relative error was found to be $1 \%$ for the lung insert $\mathrm{LN}-$ 300 , while for 8 out of the 12 reference materials, the relative error of the DECT method was below $0.43 \%$. All the relative errors can be found in the supplementary material. ${ }^{22}$ As the application of the Saito method provided satisfactory results for the prediction of $\rho_{e}$, the method was applied as such on the 3D printed samples, for which Fig. 2 visualizes the predicted values. Inspection of the Bethe-Bloch equation (1) indicates that the SPR is primarily influenced by $\rho_{e}$ and only in secondary order by $Z_{\text {eff }}$ due to the logarithmic operation on the latter. Therefore the $\rho_{e}$ estimations already suggest that different materials can be distinguished in terms of proton range shifting, namely, the significantly less than water-equivalent $\mathrm{ABS}$, the approximately water-equivalent PA-12, and the at least $9 \%$ more than water-equivalent remaining materials. Moreover, the impact of the use of additives within the same production technique (e.g., SLS) is clearly visible, as both PA-Alu and PAGF have an increased $\rho_{e}$ compared to PA-12. Caution should be exercised when interpreting the found value for ABS, as these samples were produced using a sparse filling pattern. Different values can be obtained when using a filling percentage of $100 \%$.

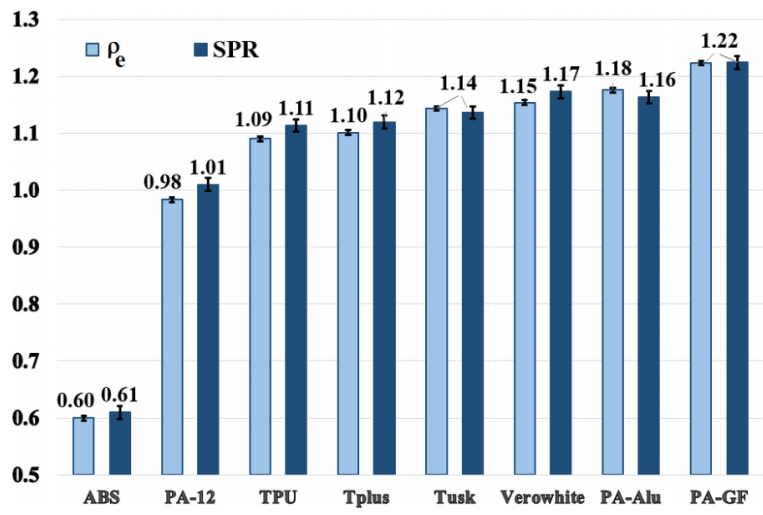

FIG. 2. Calculated $\rho_{e}$ and SPR $\left( \pm 1 S_{\text {est }}\right)$ for the 3D printed materials using the Saito respectively the Hünemohr method.

\section{C.3. Effective atomic number $Z_{\text {eff }}$}

For the reference materials, a standard error of the estimate $S_{\text {est }}$ of 0.1268 was found for $Z_{\text {eff. }}$. The largest absolute error for the reference materials was 0.32 for $\mathrm{LN}-450$, while the largest relative error was $4.67 \%$ for AP-6. For 9 out of the 12 materials, ${ }^{22}$ the absolute error was below 0.13 and the relative error below $2.16 \%$.

The calculations for $Z_{\mathrm{eff}}$ of the 3D printed samples are shown in Fig. 3. Two groups can be distinguished: the majority of the samples with a $Z_{\text {eff }}$ equal to or lower than 6.52 , and Tusk, PA-Alu, and PA-GF with a $Z_{\text {eff }}$ between 9.56 and 10.43. The $Z_{\text {eff }}$ estimations can be linked to the observed CT numbers: while the one group of materials yields a lower $\mathrm{HU}_{L}(80 \mathrm{kVp})$ than the $\mathrm{HU}_{H}(\mathrm{Sn}, 140 \mathrm{kVp})$, the other group shows the opposite trend. The significantly higher values for $\mathrm{HU}_{L}$ than for $\mathrm{HU}_{H}$ suggest the increased occurrence of the photoelectric effect at diagnostic energies for Tusk, PA-Alu, and PA-GF due to the presence of elements with higher $Z$.

\section{C.4. Stopping power ratio to water SPR}

For the reference materials, a standard error of the estimate $S_{\text {est }}$ of 0.0112 was found for the SPR. The largest relative error of $1.6 \%$ occurred for $\mathrm{LN}-300$. For 10 out of the 12 reference

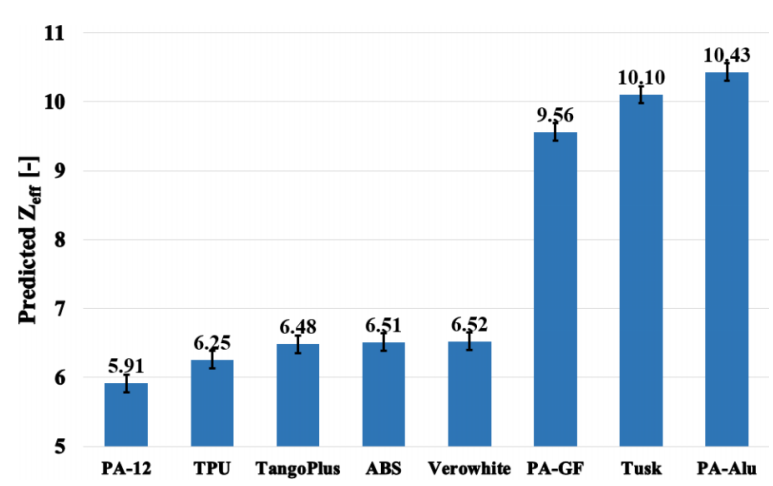

FIG. 3. Calculated $Z_{\text {eff }}\left( \pm 1 S_{\text {est }}\right)$ for the 3D printed samples using the Landry method. 
TABLE IV. Measured linear attenuation coefficients at two photon energies for the 3D printed samples and for PW.

\begin{tabular}{lllllr}
\hline \hline & \multicolumn{2}{c}{$\mu_{\mathrm{tr}}\left(\mathrm{cm}^{-1}\right)$} & & \multicolumn{2}{c}{$\mu_{\mathrm{tr}}(\%$ of PW $)$} \\
\cline { 2 - 3 } \cline { 5 - 6 } Material & $6 \mathrm{MV}$ & $10 \mathrm{MV}$ & & $6 \mathrm{MV}$ & $10 \mathrm{MV}$ \\
\hline ABS & 0.0297 & 0.0227 & 62.2 & 62.5 \\
Tusk & 0.0538 & 0.0409 & & 112.6 & 112.7 \\
TangoPlus & 0.0517 & 0.0396 & & 108.1 & 109.0 \\
VeroWhite & 0.0546 & 0.0416 & & 114.2 & 114.5 \\
PA-12 & 0.0472 & 0.0351 & & 98.8 & 96.7 \\
PA-Alu & 0.0598 & 0.0463 & & 125.1 & 127.6 \\
PA-GF & 0.0572 & 0.0446 & & 119.7 & 122.9 \\
TPU & 0.0521 & 0.0397 & & 109.1 & 109.4 \\
PW & 0.0478 & 0.0363 & 100.0 & 100.0 \\
\hline \hline
\end{tabular}

materials, ${ }^{22}$ the error was smaller than or equal to $1.04 \%$. For the 3D printed samples (see Fig. 2), the different behaviors relative to water, as already observed in the calculation of $\rho_{e}$ in Sec. 3.C.2, are confirmed.

\section{D. Beam measurements}

\section{D.1. Photon linear attenuation coefficient $\mu_{\mathrm{tr}}$}

The attenuation coefficients $\mu_{\mathrm{tr}}$ measured at the two energies are displayed in Table IV. No directional dependency was observed. For the measured $\mu_{\mathrm{tr}}$ versus the predicted $\rho_{e}$ a linear relationship $\left(R^{2}>0.95\right)$ was found, as documented in the supplementary material. ${ }^{22}$

\section{D.2. Proton range shift}

Figure 4 displays a selection of the acquired Bragg peaks, with and without 3D printed material inserted. Repeated application of the measurement method on the PW insert with known composition yielded deviations from the theoretical range shift of $\pm 0.25 \mathrm{~mm}$.

Figure 5(a) shows the error on the DECT calculated range shift for the 3D printed samples. For one sample material, Tusk, the range shift calculation differs more than $1 \mathrm{~mm}$ from the measured range shift. For seven out of eight materials, the deviation is no more than $0.66 \mathrm{~mm}$.

In Fig. 5(b) the difference in measured range shift for the two measured orientations of the samples is shown. For three

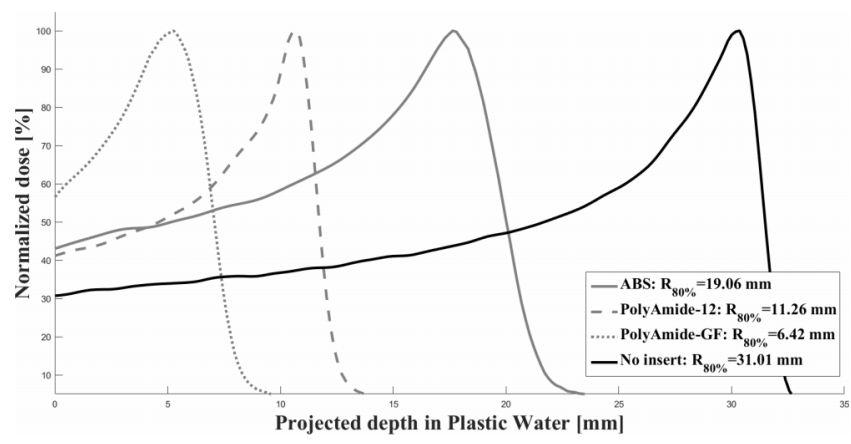

FIG. 4. Selection of acquired Bragg peaks with and without 3D printed material inserted. of the sample materials (ABS, VeroWhite and PA-12), the range shift difference between two orientations was observed to be above $0.5 \mathrm{~mm}$, which was the maximum difference between the repeated measurements on the PW sample. This suggests the existence of an orientation dependency for range shifting for these materials.

\section{DISCUSSION}

The first goal of this study was to perform a broad characterization of $3 \mathrm{D}$ printed materials for the future construction of a novel, multifunctional immobilization structure. The resulting data can be used for an optimal design of such a device in terms of structural strength, elasticity, and geometrical and radiological properties.

The wide range of Young's moduli encountered in the compression tests, from the rubberlike flexibility of TangoPlus to the polystyrenelike rigidity of Tusk and PA, makes a set of materials available to fulfill specific mechanical functionalities. The feature of high elasticity of these former materials, for instance, could be used advantageously to increase comfort in regions of elevated contact pressure. Such flexibility might also be an advantage to maintain a close fit for some parts of the immobilization in a situation with small anatomical changes. On the other hand, for regions requiring a tight tolerance on the positioning accuracy, a more rigid material should be used rather than a flexible material. For the case of a uniform load $q$ on a beam supported at both ends, with a Young's modulus $E$, a length $L$, and a square section with side a, the maximum deformation can be calculated as $\delta=5 q / 32 E(L / a)^{4}$. This simplified calculation can be applied to the structural support of a head by a beam in, for example, $3 \mathrm{D}$ printed ABS with the same dimensions as the test samples. As such, a realistic but overestimated load of $10 \mathrm{~kg}$ of a patient head would result in a deformation below $0.1 \mathrm{~mm}$, which is clearly acceptable. Obviously, such simplified calculations can only be indicative for the conception of a design. Also, the stiffness measurements were performed on standardized, simple shapes. As the design of the printed immobilization device proceeds, a necessary next step would be to assess the mechanical integrity and the deformations of more complex structures by means of experiments and/or in silico finite element method (FEM) analysis.

Besides the mentioned mechanical stiffness and deformation itself, the change in mechanical response due to the administration of clinically relevant dose levels of $70 \mathrm{~Gy}$ (multiplied with a safety factor of around 1.4) is a point of consideration as well. In this regard, the performed compression tests prior and after irradiation provide valuable information. In general, the results suggest that most of the tested materials experience a stiffening effect due to the exposure to radiation. This may be explained by a facilitation of the chain forming due to the irradiation, which could result in an increased polymerization and hence stiffening of the materials. Depending on the type of polymer and the printing process used, the susceptibility to this effect indeed may differ from one material to another. A side effect of this stiffening could be that the material becomes more brittle, meaning less 

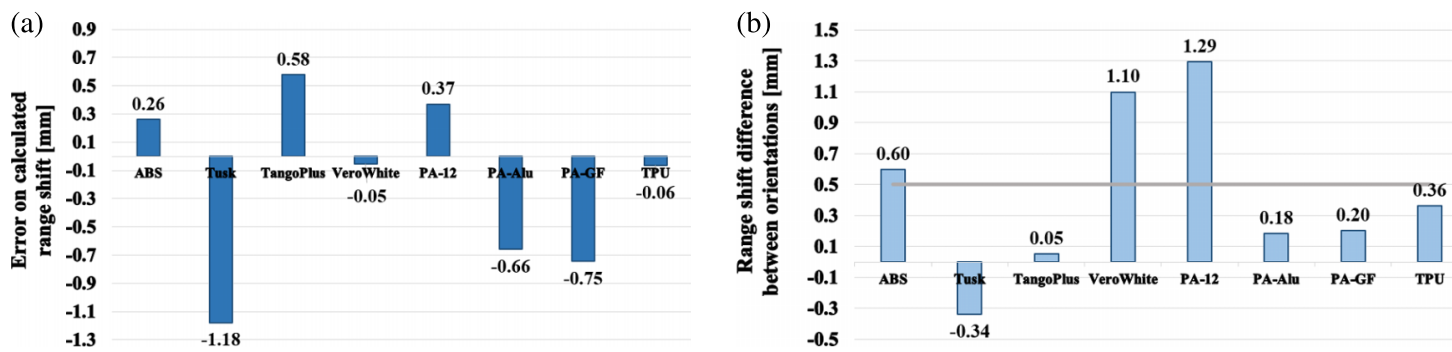

Fig. 5. (a) Error on the calculated range shift for one orientation, and (b) measured range shift difference between two orientations.

deformation can be sustained before breaking and material rupture occurs more abruptly. These results, however, may be less pronounced when using clinical dose levels without the multiplication factor. Also, as only the influence of photon irradiation on the mechanical stiffness was assessed, it might be valuable to repeat this exercise for proton irradiation, since the interaction of protons with matter yields additional processes such as nuclear interactions which might cause additional effects to occur.

The measured interaction of both photons and protons with the $3 \mathrm{D}$ printed materials allows to assess their beam modifying properties when used in an immobilization structure. The three observed degrees of water-equivalence for both types of interaction can, namely, be deliberately exploited in the design. For instance, ABS may be used for parts where minimal beam interaction is desired, such as support structures, whereas the near water-equivalent PA-12 may be used for parts where the radiological behavior of soft tissue is to be mimicked. The elevated beam interaction of the remaining materials, in various degrees compared to water, may be selected for regions where a maximum proximal dose shift for the same thickness of material is aimed for, for example, bolus. Given the extensiveness of this latter group of materials, additional material properties may be included in the selection process for bolus material, depending on the design requirements to be met such as the mechanical stiffness, the cost per unit of volume, or the DECT estimated $Z_{\text {eff }}$ to minimize the amount of scattering. However, the use of the most flexible materials in zones where a beam portal is assigned should be done with caution, given the poor agreement of the sample dimensions with the nominal dimensions and given the high degrees of curvature observed. The uncertainty on the thickness hence on the water-equivalent path length may result in dose delivery deviations from the planned dose. The reported printing inaccuracy, however, might be overcome by optimizing the used print direction. Moreover, the testing of a larger amount of samples might reveal better dimensional compliance for these materials.

The DECT based calculations of the radiological properties offer a first step toward the clinical implementation of in-house assessment of 3D printed materials for the proposed use in immobilization, which was the second goal of this study. Such an approach could offer possibilities to reduce the number of device-specific QA tests needed before clinical use. The calculated $\rho_{e}$ was (albeit indirectly) experimentally verified and appears to serve as an excellent predictor for the photon attenuation. Indeed, the governing relationships for the atomic mass cross section $\sigma_{\text {at }}$, respectively, electronic cross section $\sigma_{\text {el }}$ for the Compton effect can be written as ${ }^{35}$

$$
\left\{\begin{array}{l}
\frac{\sigma_{\mathrm{at}}}{\rho}=\frac{N_{A} Z}{A} \sigma_{\mathrm{el}} \rightarrow \sigma_{\mathrm{at}}=\rho_{e} \sigma_{\mathrm{el}} \\
\sigma_{\mathrm{el}} \propto Z^{0}
\end{array} \Rightarrow \sigma_{\mathrm{at}} \propto \rho_{e} .\right.
$$

Since at clinical MV photon energies the Compton effect dominates and $\sigma_{\text {at }}$ scales linearly with $\rho_{e}$, the observed linear variation of $\mu_{\mathrm{tr}}$ with $\rho_{e}$ is as expected. The significant improvement of the accuracy of the $\rho_{e}$ calculation ${ }^{28}$ for the reference materials in general indicates the potential added value of the DECT over the SECT base method for treatment planning dose calculations. ${ }^{36,37}$

The found uncertainty of $\pm 0.25 \mathrm{~mm}$ on the used range shift measurement method is in line with the findings of Zhao and Das. ${ }^{32}$ Given this uncertainty, in general the DECT derived SPR provides a good estimation for the range shifting of 3D printed materials. The systematically larger deviations for the higher $Z_{\text {eff }}$ materials, however, might indicate that the used parameterization $I=f\left(Z_{\text {eff }}\right)$ which is used in the SPR estimation can be improved for this group of materials. This explanation seems viable since the used parameterization was derived from a large set of tissue-equivalent materials, whereas the $3 \mathrm{D}$ printed materials that yield the larger deviations do not belong to this group since they contain elements such as $\mathrm{Si}$ and Al.

Additionally, the SPR estimation method does not allow to take into account the sample orientation dependency of the achieved range shift as observed for certain printed materials. The CT-numbers distribution, however, might hint at the presence of this effect, as some correlation was observed between the orientation dependency and the CT-numbers distribution of the ABS and VeroWhite samples, as these exhibited a relatively high skewness and kurtosis. ${ }^{22}$ Since the necessarily used tilt in the measurement setup might already have dampened or even concealed this orientation dependency, this effect might even be more pronounced when proton beam incidences perfectly parallel or orthogonal to the printed layers are used. An altered measurement setup using a Bragg peak ionization chamber to longitudinally scan the range shifted beam incidence in a water tank could provide more information on this effect, as this setup enables to measure the residual range under perfectly aligned beam incidence and with a better accuracy. In any case, the orientation dependency should be carefully considered when selecting and validating 3D printed materials for the intended multifunctional immobilization device. 


\section{CONCLUSIONS}

3D printing technology opens new possibilities to design immobilization devices which could include multiple functionalities beyond fixation only. Today, however, little is known about the relevant properties for this application. Due to the additive manufacturing process, 3D printed materials cannot be considered to exhibit the same behavior as their traditionally manufactured counterparts, which is, for instance, illustrated by the observed orientation dependency of the range shift for certain printed materials. In this study, the first step toward 3D printed, multifunctional immobilization was performed, by going through a candidate clinical workflow for the first time: from the material printing to DECT characterization with a verification through beam measurements. Besides a proof of concept for beam modification, the mechanical response of printed materials was also investigated to assess their capabilities for positioning functionality. For the studied set of printed materials and techniques, a wide variety of mechanical and radiological properties can be selected from for the intended purpose. Moreover, the elaborated hybrid DECT methods aid in performing in-house QA of 3D printed components, as these methods enable the estimation of the radiological properties relevant for use in radiation therapy.

\section{ACKNOWLEDGMENTS}

We would like to thank Walter Coudyzer and Hilde Bosmans (University Hospitals Leuven) for providing access to the DECT scanner. Wouter Crijns (University Hospitals Leuven) and Gilles Defraene (KU Leuven) are acknowledged for their support in the execution of the different experiments. The colleagues of UCL and the affiliated University Hospitals Saint-Luc are highly appreciated for various contributions, especially Nancy Postiau (UCL) for providing repeated access to the proton beam line, and Stefaan Vynckier and Antoine Delor for providing access to the Gammex Cheese Phantom. We are indebted to Eefje Verhoelst and Tom Cluckers (Materialise) for providing the 3D printed samples and for various 3D printing related support. Karin Haustermans is partly funded by the Research Foundation Flanders.

\section{CONFLICT OF INTEREST DISCLOSURE}

The authors have no COI to report.

\footnotetext{
a)Electronic mail: michiels.steven@kuleuven.be

${ }^{b)}$ Electronic mail: tom.depuydt@kuleuven.be

${ }^{1}$ A. J. Lomax, "Intensity modulated proton therapy and its sensitivity to treatment uncertainties 1: The potential effect of calculational uncertainties," Phys. Med. Biol. 53, 1027-1042 (2008).

${ }^{2}$ A. J. Lomax, "Intensity modulated proton therapy and its sensitivity to treatment uncertainties 2: The potential effects of interfraction and inter-field motions," Phys. Med. Biol. 53, 1043-1056 (2008).

${ }^{3}$ J. R. Palta and D. K. Yeung, in Proton Therapy Physics, edited by H. Paganetti (CRC, Boca Raton, 2011), pp. 413-434.
}

${ }^{4}$ M. Engelsman, M. Schwarz, and L. Dong, "Physics controversies in proton therapy," Semin. Radiat. Oncol. 23, 88-96 (2013).

${ }^{5}$ J. A. Langendijk, P. Lambin, D. De Ruysscher, J. Widder, M. Bos, and M. Verheij, "Selection of patients for radiotherapy with protons aiming at reduction of side effects: The model-based approach," Radiother. Oncol. 107, 267-273 (2013).

${ }^{6}$ B. L. T. Ramaekers, J. P. C. Grutters, M. Pijls-Johannesma, P. Lambin, M. A. Joore, and J. A. Langendijk, "Protons in head-and-neck cancer: Bridging the gap of evidence," Int. J. Radiat. Oncol., Biol., Phys. 85, 1282-1288 (2013).

${ }^{7}$ F. P. W. Melchels, J. Feijen, and D. W. Grijpma, "A review on stereolithography and its applications in biomedical engineering," Biomaterials 31, 6121-6130 (2010).

${ }^{8}$ J. S. Matsumoto, J. M. Morris, T. A. Foley, E. E. Williamson, S. Leng, K. P. McGee, J. L. Kuhlmann, L. E. Nesberg, and T. J. Vrtiska, "Threedimensional physical modelling: Applications and experience at Mayo clinic," RadioGraphics 35, 1989-2006 (2015).

${ }^{9}$ S. G. Ju, M. K. Kim, C. S. Hong, J. S. Kim, Y. Han, D. H. Choi, D. Shin, and S. B. Lee, "New technique for developing a proton range compensator with use of a 3-dimensional printer," Int. J. Radiat. Oncol., Biol., Phys. 88, 453-458 (2014).

${ }^{10}$ C. Lindsay, J. Kumlin, A. Jirasek, R. Lee, D. M. Martinez, P. Schaffer, and C. Hoehr, "3D printed plastics for beam modulation in proton therapy," Phys. Med. Biol. 60, N231-N240 (2015).

${ }^{11} \mathrm{~W}$. Zou et al., "Potential of 3D printing technologies for fabrication of electron bolus and proton compensators," J. Appl. Clin. Med. Phys. 16, 90-98 (2015).

${ }^{12}$ R. Mayer, P. Liacouras, A. Thomas, M. Kang, L. Lin, and C. B. Simone, "3D printer generated thorax phantom with mobile tumor for radiation dosimetry," Rev. Sci. Instrum. 86, 074301 (2015).

${ }^{13}$ P. H. Ahn, A. I. Ahn, C. J. Lee, J. Shen, E. Miller, A. Lukaj, E. Milan, R. Yaparpalvi, S. Kalnicki, and M. K. Garg, "Random positional variation among the skull, mandible, and cervical spine with treatment progression during head-and-neck radiotherapy," Int. J. Radiat. Oncol., Biol., Phys. 73, 626-633 (2009).

${ }^{14}$ L. Zhang, A. S. Garden, J. Lo, K. K. Ang, A. Ahamad, W. H. Morrison, D. I. Rosenthal, M. S. Chambers, X. R. Zhu, R. Mohan, and L. Dong, "Multiple regions-of-interest analysis of setup uncertainties for head-andneck cancer radiotherapy," Int. J. Radiat. Oncol., Biol., Phys. 64, 1559-1569 (2006).

${ }^{15}$ J. L. Barker, Jr. et al., "Quantification of volumetric and geometric changes occurring during fractionated radiotherapy for head-and-neck cancer using an integrated CT/linear accelerator system," Int. J. Radiat. Oncol., Biol., Phys. 59, 960-970 (2004).

${ }^{16}$ D. L. J. Barten, J. P. Tol, M. Dahele, B. J. Slotman, and W. F. A. R. Verbakel, "Comparison of organ-at-risk sparing and plan robustness for spot-scanning proton therapy and volumetric modulated arc photon therapy in head-andneck cancer,” Med. Phys. 42, 6589-6598 (2015).

${ }^{17} \mathrm{~S}$. Safai, T. Bortfeld, and M. Engelsman, "Comparison between the lateral penumbra of a collimated double-scattered beam and uncollimated scanning beam in proton radiotherapy," Phys. Med. Biol. 53, 1729-1750 (2008).

${ }^{18}$ J. M. Sisterson, M. M. Urie, A. M. Koehler, and M. Goitein, "Distal penetration of proton beams: The effects of air gaps between compensating bolus and patient," Phys. Med. Biol. 34, 1309-1315 (1989).

${ }^{19}$ U. Titt, D. Mirkovic, G. O. Sawakuchi, L. A. Perles, W. D. Newhauser, P. J. Taddei, and R. Mohan, "Adjustment of the lateral and longitudinal size of scanned proton beam spots using a pre-absorber to optimize penumbrae and delivery efficiency," Phys. Med. Biol. 55, 7097-7106 (2010).

${ }^{20}$ Materialise NV, 3D printed material properties, 2015, http://manufacturing. materialise.com/sites/default/files/public/AMS/Datasheets/ams_datasheets august2015.pdf.

${ }^{21}$ American Society for Testing and Materials, "Standard test method for compressive properties of rigid plastics," in Report D695-15 (ASTM, West Conshohocken, 2015).

${ }^{22}$ See supplementary material at http://dx.doi.org/10.1118/1.4962033 for the elemental weight fractions and other properties of the Gammex inserts and of the CIRS PW; the CT numbers of the scans of the reference materials and of the $3 \mathrm{D}$ printed samples, together with an overview of the DE-CT based estimated radiological properties; and the measured $\mu_{\text {tr }}$ versus calculated $\rho_{\mathrm{e}}$ of the 3D printed samples and of PW. 
${ }^{23}$ H. E. Johns and J. R. Cunningham, The Physics of Radiology (Charles C. Thomas, Springfield, IL, 1983).

${ }^{24}$ International Commission on Radiation Units and Measurements Report No. 49, 1993.

${ }^{25}$ S. M. Seltzer and M. J. Berger, "Evaluation of the collision stopping power of elements and compounds for electrons and positrons," Int. J. Appl. Radiat. Isot. 33, 1189-1218 (1982).

${ }^{26}$ N. Hünemohr, B. Krauss, C. Tremmel, B. Ackermann, O. Jäkel, and S. Greilich, "Experimental verification of ion stopping power prediction from dual energy CT data in tissue surrogates," Phys. Med. Biol. 59, 83-96 (2014).

${ }^{27}$ U. Schneider, E. Pedroni, and A. J. Lomax, "The calibration of CT Hounsfield units for radiotherapy treatment planning," Phys. Med. Biol. 41, 111-124 (1996).

${ }^{28} \mathrm{M}$. Saito, "Potential of dual-energy subtraction for converting CT numbers to electron density based on a single linear relationship," Med. Phys. 39, 2021-2030 (2012).

${ }^{29}$ G. Landry, J. Seco, M. Gaudreault, and F. Verhaegen, "Deriving effective atomic numbers from DECT based on a parameterization of the ratio of high and low linear attenuation coefficients," Phys. Med. Biol. 58, 6851-6866 (2013).
${ }^{30}$ R. A. Rutherford, B. R. Pullan, and I. Isherwood, "Measurement of effective atomic number and electron density using an EMI scanner," Neuroradiology 11, 15-21 (1976).

${ }^{31}$ D. Georg and A. Dutreix, "Methods for beam data acquisition offered by a mini-phantom," Phys. Med. Biol. 44, 817-832 (1999).

${ }^{32}$ L. Zhao and I. J. Das, "Gafchromic EBT film dosimetry in proton beams," Phys. Med. Biol. 55, N291-N301 (2010).

${ }^{33}$ W. Crijns, F. Maes, U. A. van der Heide, and F. Van den Heuvel, "Calibrating page sized Gafchromic EBT3 films,” Med. Phys. 40, 012102 (13pp.) (2013).

${ }^{34}$ B. Gottschalk, in Proton Therapy Physics, edited by H. Paganetti (CRC, Boca Raton, 2011), p. 32.

${ }^{35}$ F. H. Attix, Introduction to Radiological Physics and Radiation Dosimetry (Wiley-VCH, Weinheim, 1986).

${ }^{36}$ M. Bazalova, J. F. Carrier, L. Beaulieu, and F. Verhaegen, "Dual-energy CT-based material extraction for tissue segmentation in Monte Carlo dose calculations," Phys. Med. Biol. 53, 2439-2456 (2008).

${ }^{37}$ N. Hünemohr, H. Paganetti, S. Greilich, O. Jäkel, and J. Seco, "Tissue decomposition from dual energy CT data for MC based dose calculation in particle therapy," Med. Phys. 41, 061714 (14pp.) (2014). 